\title{
Transmit and Receive Array Structure Design of Two-Dimensional hybrid Phased-MIMO Radar based on Nested Array
}

\author{
Tianhao CHENG ${ }^{1, a}$, Buhong Wang ${ }^{1}$,Qiaoge Liu ${ }^{1}$, Jiwei Tian ${ }^{1}$ \\ ${ }^{1}$ College of Information and Navigation, Air Force Engineering University, Xi'an 710077, China
}

\begin{abstract}
In order to reduce the loss of Degree of Freedom (DOF) brought by the transmit subarray splitting of twodimensional hybrid phased-MIMO radar, this paper presents a design method of transmitting and receiving array based on nested array structure. Firstly, a two-dimensional hybrid phased-MIMO radar transmitting array based on one-dimensional nested array is presented. On this basis, the receiving end is set as a nested array, and finally a virtual array and difference coarray are formed to expand the number of virtual array elements. The expansion increases the DOF of arrays while preserving the advantages of hybrid phased-MIMO radars. Simulation experiments show that compared with the traditional and coprime hybrid phased-MIMO radar, the proposed method can effectively improve the array DOF and Direction-of-Arrival (DOA) estimation accuracy.
\end{abstract}

\section{Introduction}

The hybrid phased-multiple-input multiple-output (MIMO) technology of array antennas has been widely used in the field of communication in recent years [1]. It has a good prospect in application[2-3].

However, although hybrid phased-MIMO radar has the above advantages, both gain levels are somewhat weakened compared to conventional MIMO radars and phased array radars. In particular, the subarray splitting of the transmitting end causes loss of partial waveform diversity gain while obtaining the transmit coherent processing gain, and the virtual aperture of the radar system is lost, so the DOF is reduced inevitably. therefore, the array's DOA estimation performance worse.

In order to make up for the aperture loss caused by subarray separation, many experts and scholars have done considerable works. Although the MRA(Minimum Redundant Array) proposed in [4] can extend the array aperture to some extent, the optimal search algorithm for constructing arrays brings higher computational complexity and no closed expression. The coprime array proposed in [5] can implementing DOF expansion of $O(M P)$ with $M+P$ array elements under the premise of the limited number of actual array elements and less computation, but the difference coarray of coprime array is not "hole-free", which has a certain impact on the realization of radar parameter estimation, and wastes part of the virtual aperture. In response to this problem, the nested array proposed in [4] can form a DOF extension of $O\left(Q^{4}\right)$ by using only $Q$ array elements without increasing the number of actual array elements by constructing a "hole-free" difference coarray. The relationship between aperture expansion and computational complexity is effectively balanced, and the implementation of radar parameter estimation algorithm is facilitated.

In view of the many advantages of nested arrays, many scholars have combined nested arrays with MIMO radar design and conducted related research. In [5], based on the nested array structure, multiple subarrays are divided from the nested array, the waveforms of the subarrays are orthogonal, and the subarray works inside the phased array mode to realize the performance compromise between phased array and MIMO inside the nested array. In [8], nested arrays are placed on the MIMO radar transceivers, which greatly expands the array aperture and DOF. In [11], on the hybrid phasedMIMO radar system, the receiver is designed as a nested structure, combining the advantages of hybrid phasedMIMO array and nested array. However, the above studies are all directed to one-dimensional radar arrays, which cannot be fully extended to two-dimensional practical applications. In [10], this method is extended to two-dimensional hybrid phased-MIMO radar and twodimensional nested array, which expands the DOF of the two-dimensional array and improves the radar DOA estimation performance to some extent. However, the above method is only a simple design of the hybrid phased-MIMO radar receiving array, and no effective design method is proposed for the transmitting array that determines the radar performance. The transmit-end structure is the key factor determining the waveform diversity gain and transmit coherent gain of the hybrid phased-MIMO radar, which affects the radar parameter estimation performance to some extent. So optimizing the performance of hybrid phased-MIMO radar transmit

\footnotetext{
a Corresponding author: m18691805592@163.com
} 
array is a key problem that needs to be solved to limit the performance of two-dimensional hybrid phased-MIMO radar. Therefore, the nested array design of the hybrid phased-MIMO radar transmitting and receiving end is carried out. Based on the advantages of the twodimensional hybrid phased-MIMO radar, the virtual array aperture and DOF expansion are realized through nested arrays. The aperture loss caused by the splitting of the subarray is improved, and the radar parameter estimation performance is improved as well.

\section{One-dimensional nested array model}

Assuming that a nested array has $N$ array elements and $x_{i}$ represents the $i$-th array element, then the definition set $D=\left\{x_{i}-x_{j}\right\}, \quad i, j=1,2, \cdots, N \quad$ is the difference coarray of the nested array.

With the traditional $N$-element ULA, only $N$ targets in space can be identified, that is, only $N$ DOFs are owned. However, for the $N$-element one-dimensional nested array, by forming the difference coarray, the array DOF can be greatly expanded, and $D O F_{\max }$ can be used to represent the maximum DOF that the nested array can achieve, then $D O F_{\max }=N(N-1)+1$.

We assume a one-dimensional co-located MIMO radar system. The transmit and receive arrays are nested arrays with $M$ and $P$ array elements, respectively, represented by sets $\left\{T_{m}\right\}_{m=1}^{M}$ and $\left\{R_{p}\right\}_{p=1}^{P}$. Each transmit array element emits mutually orthogonal waveforms. Performing matching filtering at the receiving end, a virtual array containing $M \cdot P$ array elements can be obtained, and the position of the array elements can be expressed as:

$$
\left\{u_{k}\right\}=\left\{T_{m}+R_{p} \mid m=1,2, \cdots, M ; p=1,2, \cdots, P\right\}
$$

Where $k=1,2, \cdots, M P$, as can be seen from the above formula, the virtual array is generated by the crossaddition of the positions of the transmitting array and the receiving array elements. In order to obtain the difference coarray, it is necessary to make the virtual arrays perform the difference between the positions of the array elements, that is, $Q$, and expand to be expressed as:

$$
\begin{array}{r}
\left\{u_{k}-u_{k^{\prime}}\right\}=\left\{T_{m}+R_{p}-T_{m^{\prime}}+R_{p^{\prime}} \mid m, m^{\prime}=1,2, \cdots, M ;\right. \\
\left.p, p^{\prime}=1,2, \cdots, P\right\}
\end{array}
$$

In order to further increase the DOF and obtain a "hole-free" difference coarray, the position of the array at the transmitting end and the receiving end should satisfy the constraint:

$$
\begin{aligned}
& \max _{\left\{T_{m}\right\},\left\{R_{n}\right\}} L \quad \text { s.t. }\left|T_{m}\right|=M,\left|R_{n}\right|=P, \\
& \left\{T_{m}+R_{p}-T_{m^{\prime}}+R_{p^{\prime}}\right\} \supset\{-L, \cdots,-1,0,1, \cdots, L\}
\end{aligned}
$$

Where $L$ is the largest continuous unbroken aperture in the resulting difference coarray, ie the "hole-free" difference coarray. Among them, the difference coarray is the essence of virtual matrix expansion generated by MRA, coprime array and nested array, but the difference coarray generated by MRA and coprime array is not "hole-free", only nested array can form the largest "holefree" difference coarray. Therefore, our proposed hybrid MIMO nested array radar scheme is based on nested arrays.

\section{Two-dimensional hybrid phased-MIMO radar model}

Hybrid phased-MIMO radar is based on MIMO radar, and reasonable subarray is divided, so that the subarray works in phased array mode, and the MIMO radar state is maintained between subarrays. Considering that the transmitting end is composed of $M \times N$ array elements, the spacing of adjacent array elements is half wavelength, and is divided into $\mathrm{K}$ subarrays. The definition matrix $\boldsymbol{Z}_{k}$ is an $M \times N$-dimensional matrix containing only 0,1 elements, $M, N$ are the number of array elements per row and each column, respectively, 0 means that there is no array element at the corresponding position of the array, and 1 means the opposite meaning. Therefore, the steering vector of transmit array of the kth subarray can be expressed as:

$$
\begin{aligned}
& \boldsymbol{a}_{k}(\theta, \phi)=\operatorname{vec}\left(\boldsymbol{Z}_{\boldsymbol{k}} \odot\left[\boldsymbol{\mu}(\theta, \phi) \boldsymbol{v}^{\mathrm{T}}(\theta, \phi)\right]\right) \\
& k=1,2, \cdots, K
\end{aligned}
$$

Where: $K \leq M N, \quad \operatorname{vec}(\cdot)$ represents the vectorization of the matrix, $(\bullet)^{\mathrm{T}}$ represents the conjugate transpose, and $\odot$ represents the Hadamard product.

$$
\begin{aligned}
& \boldsymbol{\mu}(\theta, \phi)=\left[1, \mathrm{e}^{\mathrm{j} 2 \pi d_{m} \sin (\theta) \cos (\phi)}, \cdots, \mathrm{e}^{\mathrm{j} 2 \pi(M-1) d_{m} \sin (\theta) \cos (\phi)}\right]^{\mathrm{T}} \\
& \boldsymbol{v}(\theta, \phi)=\left[1, \mathrm{e}^{\mathrm{j} 2 \pi d_{n} \sin (\theta) \sin (\phi)}, \cdots, \mathrm{e}^{\mathrm{j} 2 \pi(N-1) d_{n} \sin (\theta) \sin (\phi)}\right]^{\mathrm{T}}
\end{aligned}
$$

Where $d_{m}$ and $d_{n}$ represent the spacing of adjacent array elements per row, respectively, and $\varphi$ and $\theta$ are azimuth and elevation angles.

Assuming that $w_{k}, k=1,2, \cdots K$ is the transmit weight matrix of the first subarray, the $K \times 1$-dimensional transmit coherent processing gain and the waveform diversity gain can be expressed as:

$$
\begin{gathered}
\boldsymbol{c}(\theta, \phi)=\left[w_{1}^{H} a_{1}(\theta, \phi), \cdots, w_{K}^{H} a_{K}(\theta, \phi)\right]^{\mathrm{T}} \\
\boldsymbol{d}(\theta, \phi)=\left[e^{-j \tau_{1}(\theta, \phi)}, \cdots, e^{-j \tau_{K}(\theta, \phi)}\right]^{\mathrm{T}}
\end{gathered}
$$

Where $\tau_{k}(\theta, \phi)$ is the time difference between the first subarray and the first array of the first subarray. Therefore the array manifold of the system can be expressed as:

$$
\boldsymbol{u}(\theta, \phi)=\boldsymbol{c}(\theta, \phi) \odot \boldsymbol{d}(\theta, \phi) \otimes \boldsymbol{b}(\theta, \phi)
$$

Where $\boldsymbol{b}(\theta, \phi)$ is the reception steering vector and $\otimes$ is the Kronecker product. The set of orthogonal waveforms generated by the transmitting end is $\boldsymbol{\varphi}(t)=\left[\varphi_{1}(t), \varphi_{2}(t), \cdots, \varphi_{K}(t)\right], \quad \varphi_{k}(t)=\sqrt{1 / T_{0}} e^{j 2 \pi\left(k / T_{0}\right) t}$, then the target reflected signal can be expressed as: 


$$
r(t, \theta, \phi)=\sqrt{M N / K} \beta \sum_{k=1}^{K} \boldsymbol{w}_{k}^{H} \boldsymbol{a}_{k}(\theta, \phi) \mathrm{e}^{-\mathrm{j} \tau_{k}(\theta, \phi)} \varphi_{k}(t)
$$

Therefore, the receiving end receives the signal as:

$$
\boldsymbol{x}(t)=r(t, \theta, \phi) \boldsymbol{b}(\theta, \phi)+\boldsymbol{n}(t)
$$

Where $\boldsymbol{n}(t)$ is the noise matrix, and the data matrix obtained after matched filtering is:

$$
\boldsymbol{y}=\int_{T_{0}} \boldsymbol{x}(t) \boldsymbol{\varphi}^{*}(t) d t=\sqrt{M N / K} \beta \boldsymbol{u}(\theta, \phi)+\hat{\boldsymbol{n}}
$$

Where $T_{0}$ represents the duration of a radar pulse, $\hat{\boldsymbol{n}}=\int_{T_{0}} \boldsymbol{n}(t) \boldsymbol{\varphi}^{*}(t) d t$.

\section{Nested two-dimensional hybrid phased-MIMO radar}

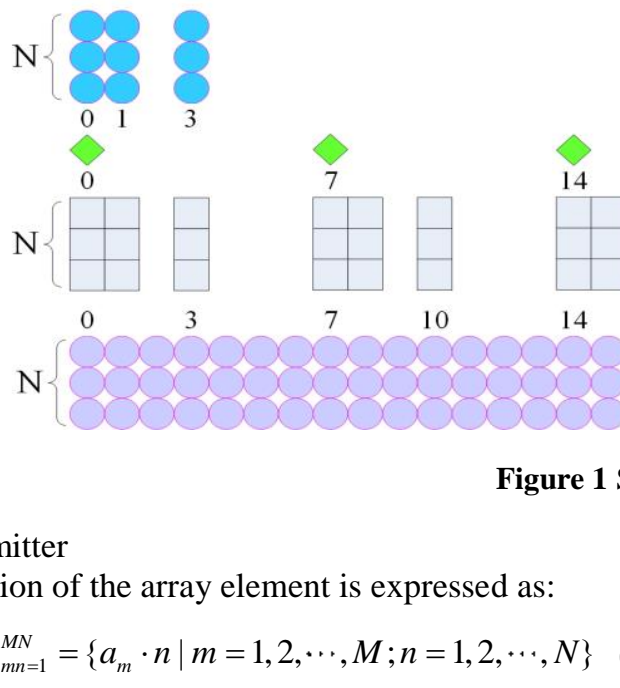

1) Transmitter

$$
\left\{T_{m, n}\right\}_{m n=1}^{M N}=\left\{a_{m} \cdot n \mid m=1,2, \cdots, M ; n=1,2, \cdots, N\right\}
$$

Where $\left\{a_{m}\right\}_{m=1}^{M}$ represents the position of the array element of the horizontal nested array, and $\mathrm{n}$ represents the position of the array element of the phased array arranged in the longitudinal direction. The number of DOF and array elements satisfy the following relationship:

$$
f_{M}=\left\{\begin{array}{l}
M^{2} / 2+M-1, \text { When } \mathrm{M} \text { is even } \\
(M+1)^{2} / 2-1, \text { When } \mathrm{M} \text { is odd }
\end{array}\right.
$$

2) Receiver

The receiving end is composed of another nested array, but its array spacing is expanded by $S$ times compared to the nested array at the transmitting end:

$$
\left\{R_{p}\right\}_{p=1}^{P}=\left\{b_{p} \cdot S \mid p=1,2, \cdots, P\right\}
$$

Where $\left\{b_{p}\right\}_{p=1}^{P}$ is the sequence of positions of the standard nested array.

Virtual array can be obtained:

$$
\begin{aligned}
\left\{T_{m, n}+R_{p}\right\}= & \left\{a_{m} \cdot n+b_{p} \cdot S \mid m=1,2, \cdots, M ;\right. \\
& n=1,2, \cdots, N ; p=1,2, \cdots, P\}
\end{aligned}
$$

Then the difference coarray of virtual arrays can be written as:

\subsection{Array structure}

The system we constructed is shown in Figure 1. The position of the array elements is represented by sets $\left\{T_{m, n}\right\}_{m n=1}^{M N}$ and $\left\{R_{p}\right\}_{p=1}^{P}$. The transmitting end is a twodimensional plane array with the number of elements $M N$. The array is divided into $M$ subarrays, and the vertical column is a subarray. Each subarray contains $N$ array elements. If the subarrays do not overlap, they can be divided into $M$ subarrays whose waveforms are orthogonal to each other. Meanwhile, $M$ mutually orthogonal sub-arrays satisfy the positional relationship of the nested arrays to form a second-level nested array. The receiver is designed as a regular secondary nested MIMO array. The specific design is described as follows:

Transmitter

Receiver

Virtual Array

Difference Coarray

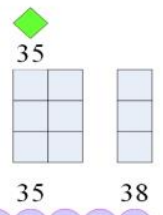

17

38

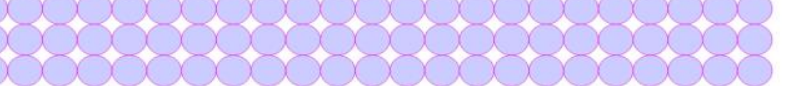


reflection coefficient vector, $J$ is the target number, then the expression of the reception covariance matrix is:

$$
\begin{aligned}
& \boldsymbol{R}_{\boldsymbol{y} \boldsymbol{y}}=E\left[\tilde{\boldsymbol{y}} \tilde{\boldsymbol{y}}^{\mathrm{H}}\right] \\
& =E\left[(\boldsymbol{U} \boldsymbol{\beta}+\sqrt{K / M N} \hat{\boldsymbol{n}})(\boldsymbol{U} \boldsymbol{\beta}+\sqrt{K / M N} \hat{\boldsymbol{n}})^{\mathrm{H}}\right]= \\
& E\left[(\boldsymbol{U} \boldsymbol{\beta}+\sqrt{K / M N} \hat{\boldsymbol{n}})\left(\boldsymbol{\beta}^{\mathrm{H}} \boldsymbol{U}^{\mathrm{H}}+\sqrt{K / M N} \hat{\boldsymbol{n}}^{\mathrm{H}}\right)\right] \\
& =\boldsymbol{U}\left[\begin{array}{cccc}
\sigma_{1}^{2} & & & \\
& \sigma_{2}^{2} & & \\
& & \ddots & \\
& & & \sigma_{J}^{2}
\end{array}\right] \boldsymbol{U}^{\mathrm{H}}+K / M N \cdot \sigma_{n}^{2} \boldsymbol{I}
\end{aligned}
$$

Where $\sigma_{n}^{2}$ is the noise variance, $E[\cdot]$ is the desired operation, $\sigma_{i}^{2}, i=1,2, \cdots, J$ is the target power. The covariance matrix in equation (18) is expanded in columns and converted into column vector form:

$$
\begin{aligned}
& \hat{\boldsymbol{y}}=\operatorname{vec}\left(\boldsymbol{R}_{\tilde{\mathbf{y}} \boldsymbol{y}}\right)=\operatorname{vec}\left[\sum_{d=1}^{D} \sigma_{d}^{2} \boldsymbol{u}\left(\theta_{d}, \phi_{d}\right) \boldsymbol{u}\left(\theta_{d}, \phi_{d}\right)^{\mathrm{H}}\right] \\
& +\sqrt{K / M N} \sigma_{n}^{2} \tilde{\boldsymbol{I}}_{n}=\left(\boldsymbol{U}^{*} \oplus \boldsymbol{U}\right) \boldsymbol{p}+\sqrt{K / M N} \sigma_{n}^{2} \overrightarrow{\boldsymbol{I}}_{n}
\end{aligned}
$$

element is 1 , the other elements are all 0 column vectors, and $\oplus$ represents the Khatri Rao product.

Bring in (8), you can get:

$$
\begin{aligned}
& \boldsymbol{u}\left(\theta_{k}, \varphi_{k}\right)=\left[\begin{array}{c}
\sqrt{M N-K+1} \\
\sqrt{M N-K+1} \\
\vdots \\
\sqrt{M N-K+1}
\end{array}\right] \odot\left[\begin{array}{c}
e^{-j \frac{2 \pi}{\lambda} u_{k}^{\mathrm{T}} N_{T 1}} \\
e^{-j \frac{2 \pi}{\lambda} u_{k}^{\mathrm{T}} N_{T 2}} \\
\vdots \\
e^{-j \frac{2 \pi}{\lambda} u_{k}^{\mathrm{T}} N_{T K}}
\end{array}\right] \otimes\left[\begin{array}{c}
e^{-j \frac{2 \pi}{\lambda} u_{k}^{\mathrm{T}} N_{R 1}} \\
e^{-j \frac{2 \pi}{\lambda} u_{k}^{\mathrm{T}} N_{R 2}} \\
\vdots \\
e^{-j \frac{2 \pi}{\lambda} u_{k}^{\mathrm{T}} N_{R P}}
\end{array}\right] \\
& =\sqrt{M N-K+1} \cdot\left[e^{-j \frac{2 \pi}{\lambda} u_{k}^{\mathrm{T}}\left(N_{T 1}+N_{R 1}\right)}, \cdots, e^{-j \frac{2 \pi}{\lambda} u_{k}^{\mathrm{T}}\left(N_{T 1}+N_{R P}\right)}, \cdots,\right. \\
& e^{-j \frac{2 \pi}{\lambda} u_{k}^{\mathrm{T}}\left(N_{T 2}+N_{R 1}\right)}, \cdots, e^{-j \frac{2 \pi}{\lambda} u_{k}^{\mathrm{T}}\left(N_{T 2}+N_{R P}\right)}, \cdots, e^{-j \frac{2 \pi}{\lambda} u_{k}^{\mathrm{T}}\left(N_{T K}+N_{R 1}\right)}, \\
& \left.\cdots, e^{-j \frac{2 \pi}{\lambda} u_{k}^{\mathrm{T}}\left(N_{T K}+N_{R P}\right)}\right]^{\mathrm{T}}
\end{aligned}
$$

Definite then the array manifold matrix without the difference coarray expansion is $\boldsymbol{U}$, then the new array manifold matrix extended by the difference coarray is defined as $\boldsymbol{A}=\boldsymbol{U}^{*} \oplus \boldsymbol{U}$, then:

Where $p=\left[\begin{array}{llll}\sigma_{1}^{2} & \sigma_{2}^{2} & \cdots & \sigma_{J}^{2}\end{array}\right]$ is the power set of $J$ targets, $\ddot{\boldsymbol{i}}=\left[e_{1}^{\mathrm{T}}, e_{2}^{\mathrm{T}}, \cdots, e_{K}^{\mathrm{T}}\right]$, where $e_{i}^{\mathrm{T}}$ represents the $\mathrm{i}$-th

$$
\boldsymbol{A}=\boldsymbol{U}^{*} \oplus \boldsymbol{U}=(M N-K+1)\left[\begin{array}{cccccc}
e^{-j \frac{2 \pi}{\lambda} u_{1}^{\mathrm{T}}\left(N_{T 1}+N_{R 1}-N_{T 1}-N_{R 1}\right)} & \cdots & e^{-j \frac{2 \pi}{\lambda} u_{i}^{\mathrm{T}}\left(N_{T 1}+N_{R 1}-N_{T 1}-N_{R 1}\right)} & \cdots & e^{-j \frac{2 \pi}{\lambda} u_{J}^{\mathrm{T}}\left(N_{T 1}+N_{R 1}-N_{T 1}-N_{R 1}\right)} \\
\vdots & \cdots & \vdots & \cdots & \vdots \\
e^{-j \frac{2 \pi}{\lambda} u_{1}^{\mathrm{T}}\left(N_{T K}+N_{R P}-N_{T 1}-N_{R 1}\right)} & \cdots & e^{-j \frac{2 \pi}{\lambda} u_{i}^{\mathrm{T}}\left(N_{T K}+N_{R P}-N_{T 1}-N_{R 1}\right)} & \cdots & e^{-j \frac{2 \pi}{\lambda} u_{J}^{\mathrm{T}}\left(N_{T K}+N_{R P}-N_{T 1}-N_{R 1}\right)} \\
\vdots & \cdots & \vdots & \cdots & \vdots \\
e^{-j \frac{2 \pi}{\lambda} u_{1}^{\mathrm{T}}\left(N_{T 1}+N_{R 1}-N_{T K}+N_{R P}\right)} & \cdots & e^{-j \frac{2 \pi}{\lambda} u_{i}^{\mathrm{T}}\left(N_{T 1}+N_{R 1}-N_{T K}+N_{R P}\right)} & \cdots & e^{-j \frac{2 \pi}{\lambda} u_{J}^{\mathrm{T}}\left(N_{T 1}+N_{R 1}-N_{T K}+N_{R P}\right)} \\
\vdots & \cdots & \vdots & \cdots & \vdots \\
e^{-j \frac{2 \pi}{\lambda} u_{1}^{\mathrm{T}}\left(N_{T K}+N_{R P}-N_{T K}-N_{R P}\right)} & \cdots & e^{-j \frac{2 \pi}{\lambda} u_{i}^{\mathrm{T}}\left(N_{T K}+N_{R P}-N_{T K}-N_{R P}\right)} & \cdots & e^{-j \frac{2 \pi}{\lambda} u_{J}^{\mathrm{T}}\left(N_{T K}+N_{R P}-N_{T K}-N_{R P}\right)}
\end{array}\right]_{K^{2} P^{2} \times J}
$$

After the manifold matrix is multiplied by Khatri-Rao, the position of the array elements of the difference coarray is subtracted, and a new manifold matrix of $K^{2} P^{2} \times J$-dimension is formed. At this time, the matrix dimension has been greatly increased compared with the old manifold matrix. However, since there are many repeated elements in these row vectors, it is necessary to de-reduce the new manifold matrix. Suppose $M$ is an odd number and $P$ is an even number. The matrix defined for $\boldsymbol{A}$ de-redundant is $\boldsymbol{A}_{1}$, which contains the number of elements of the manifold matrix as $\left[(M+1)^{2} / 2-1\right] \cdot\left[P^{2} / 2+P-1\right] \cdot J=f \cdot J$, where $f$ is the number of rows of the manifold matrix after deduplication, $J$ is The number of columns in the manifold matrix. Therefore, the corresponding $\boldsymbol{y}$ after removing the corresponding row becomes:

$$
\boldsymbol{y}_{1}=\boldsymbol{A}_{1} p+\frac{K}{M N} \sigma_{n}^{2} \overrightarrow{\mathrm{e}}
$$

Among them, $\overrightarrow{\mathrm{e}} \in \mathbf{R}^{((f) \times 1)}$ is a vector whose all points are all 0 except that point $(f+1) / 2$ is 1 . It can be seen that the $y_{1}$ dimension after de-redundancy is greatly increased compared to formula (17), which is the essence of the increase in array DOF formed by our proposed method.

Next, the manifold matrix is divided into $(f+1) / 2$ fully overlapping subarrays according to the number of rows, each subarray containing $(f+1) \cdot J / 2$ elements, wherein the position of the number of rows of the i-th subarray is:

$$
\{(-i+1+n), n=0,1, \cdots,(f-1) / 2\}
$$

Then, correspondingly, the number of rows of the i-th subarray corresponds to the $(f+1) / 2-i+1$-th row to the $f-i+1 \quad$-th row in $y_{1}$. Define $\boldsymbol{\Phi}=\operatorname{diag}\left(e^{-j(2 \pi / \lambda) d u_{1}}, e^{-j(2 \pi / \lambda) d u_{2}}, \cdots, e^{-j(2 \pi / \lambda) d u_{J}}\right)$, where, $\mathrm{d}$ is a distance of one and a half wavelengths. Take the $(f+1) / 2$-dimensional matrix from the $(f+1) / 2-i+1$ - 
th line to the $(f+1)-i+1$-th line of $\boldsymbol{A}_{1}$, which is defined as $\boldsymbol{A}_{1 \mathrm{i}} \cdot \mathrm{e}_{i}^{\prime}$ is a column vector whose value is all 0 except for the i-th point. Then according to the literature [4] can get:

$$
\mathbf{y}_{1 \mathrm{i}}=\mathrm{A}_{1 \mathrm{i}} \Phi^{i-1} \mathrm{p}+\frac{K}{M N} \sigma_{n}^{2} \mathrm{e}_{i}
$$

Therefore, you can get:

$$
\begin{aligned}
& \boldsymbol{R}_{\mathrm{i}} \triangleq E\left\{\boldsymbol{y}_{1 \mathrm{i}} \boldsymbol{y}_{1 \mathrm{i}}^{\mathrm{H}}\right\}=\boldsymbol{A}_{11} \boldsymbol{\Phi}^{i-1} \mathrm{pp}^{\mathrm{H}}\left(\boldsymbol{\Phi}^{i-1}\right)^{\mathrm{H}} \boldsymbol{A}_{11}{ }^{\mathrm{H}} \\
& +\frac{K^{2}}{M^{2} N^{2}} \sigma_{n}^{2} \mathrm{e}_{i} \mathrm{e}_{i}{ }^{\mathrm{H}}+\frac{K}{M N} \boldsymbol{A}_{11} \boldsymbol{\Phi}^{i-1} \mathrm{pe}_{i}{ }^{\mathrm{H}} \\
& +\frac{K}{M N} \sigma_{n}^{2} \mathrm{e}_{i} \mathrm{p}^{\mathrm{H}}\left(\boldsymbol{\Phi}^{i-1}\right)^{\mathrm{H}} \boldsymbol{A}_{11}{ }^{\mathrm{H}}
\end{aligned}
$$

For averaging different $i$, the covariance matrix after spatial smoothing:

$$
\boldsymbol{R}_{\mathrm{ss}}=\frac{1}{(f+1) / 2} \sum_{i=1}^{(f+1) / 2} \boldsymbol{R}_{\mathrm{i}}
$$

It can be applied to DOA estimation.

\subsection{Performance analysis}

The example given is shown in Figure 1. The transmitting end has three array elements in the horizontal direction, $N=20$ in the vertical direction (only three are drawn in the figure), and four array elements in the receiving end. Because of the symmetry of the position of the array element, we only draw a non-negative part in the difference coarray. According to the foregoing, since the horizontal aperture of the array of the difference of the array is 7, the expansion ratio of the array element at the receiving end is $S=7$. Therefore, the difference coarray formed by the virtual array is a "hole-free" twodimensional array.

The two-dimensional hybrid phased-MIMO radar is similar to our proposed hybrid phased-MIMO radar. The difference is that the nested array arrangement in the horizontal direction of the transceiver becomes a coprime arrangement. In order to make the comparison clearer, we chose to use the coprime array with closed expression proposed in [11]. In the lateral direction, the coprime arrays consists of a pair of ULAs having $M_{c} \cdot \lambda / 2$ and $N_{c} \cdot \lambda / 2$ cell spacings, where $\lambda$ is the signal wavelength and $M_{c}$ and $P_{c}$ are the coprime integers.

At this time, the relationship between the number of elements in the one-dimensional coprime arrays obeys the following relationship: $2 M_{c}+P_{c}-1=Q$, and requires $M_{c} 、 P_{c}$ to be coprime, and the values of $2 M_{c}-1$ and $P_{c}$ should be similar, so as to ensure the maximum DOF of the coprime array. According to [11], the maximum DOF of the coprime arrays, $D O F_{c \max }=\left\{\left(7 M_{c}-3\right) P_{c}+M_{c}\right\} \cdot N$. Wherein, $M_{c}, P_{c}$, and $D O F_{c}$ respectively represent the horizontal array element number of the transmitting end of the coprime array, the number of receiving end array elements, and the representative nested array corresponding to DOF, $M_{n}, \quad P_{n}$ and $D O F_{n}$.

In order to satisfy the condition of coprime, the values of $2 M_{c}-1$ and $P_{c}$ are not approximately equal. In order to compare the best performance of the coprime array and the nested array, we construct a $Q$ value that satisfies $2 M_{c}-1=P_{c}$ and then compare them:

Table 1 DOF of discontinuous $Q$ value

\begin{tabular}{cccccc}
\hline$Q$ & 10 & 18 & 26 & 34 & 42 \\
\hline$M_{c}$ & 3 & 5 & 7 & 9 & 11 \\
$P_{c}$ & 5 & 9 & 13 & 17 & 21 \\
$D O F_{c}$ & $93 \mathrm{~N}$ & 293 & 605 & 1029 & 1565 \\
& & $\mathrm{~N}$ & $\mathrm{~N}$ & $\mathrm{~N}$ & $\mathrm{~N}$ \\
$M_{n}$ & 5 & 9 & 13 & 17 & 21 \\
$P_{n}$ & 5 & 9 & 13 & 17 & 21 \\
$D O F_{n}$ & 289 & 2401 & 9409 & 2592 & 5808 \\
& $\mathrm{~N}$ & $\mathrm{~N}$ & $\mathrm{~N}$ & $1 \mathrm{~N}$ & $1 \mathrm{~N}$ \\
\hline
\end{tabular}

It can be seen from Table 1 that the DOF of the coprime hybrid phased-MIMO radar still lags behind the nested hybrid phased-MIMO radar, despite the optimal arrangement of the coprime array. This is because $D O F_{c \max }$ can only implement the DOF of $O\left(Q^{2}\right) \cdot N$, and the nested hybrid phased-MIMO radar can achieve $O\left(Q^{4}\right) \cdot N$.

\section{Simulation Results}

We use computer simulation to verify the performance of traditional, coprime and nested hybrid phased-MIMO radars.

Consider an example, shown in Figure 1, where $M_{o}=3, P_{o}=4, M_{c}=3, \quad P_{c}=2, \quad M_{n}=3$, $P_{n}=4$, take $N=20$, and detect 36 incoherent targets in space, their coordinates are evenly spaced at azimuth 20-70 degrees, pitch angle In the square area of 20-70 degrees. 2-D MUSIC spectra were estimated for these targets using a hybrid phased-MIMO radar and a nested hybrid phased-MIMO radar, respectively. The results are shown in Figure 2:

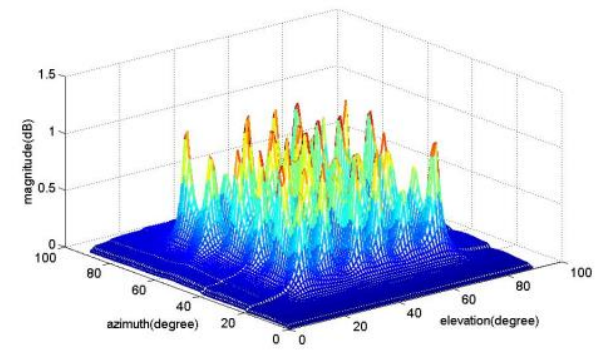

(a) MUSIC spectrum of traditional two-dimensional hybrid phased-MIMO radar 


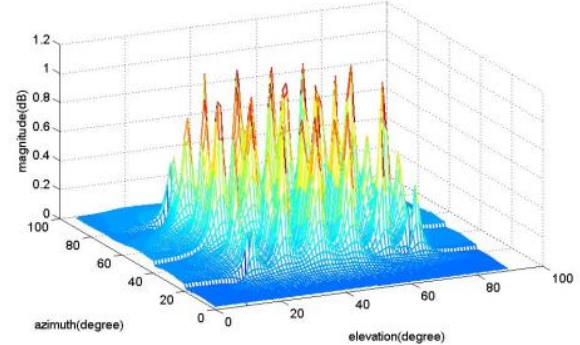

(b) MUSIC spectrum of coprime two-dimensional hybrid phased-MIMO radar

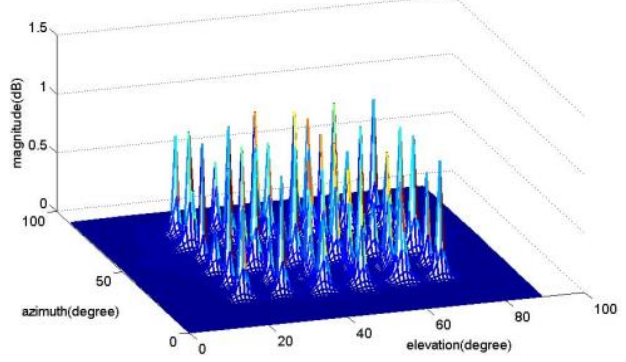

(c) MUSIC spectrum of nested two-dimensional hybrid phased-MIMO radar

Figure 2 Three types two-dimensional hybrid phased-MIMO radar MUSIC spectrum

It can be seen from the three-dimensional MUSIC spectrum of Fig. 2 that the spectral peaks of the conventional two-dimensional hybrid phased-MIMO radar are very unclear, and some degree of aliasing and distortion occur, and the effect of detecting targets is poor; Coprime hybrid phased-MIMO radar has a better MUSIC peak, but a few estimated peaks deviate from the correct position; and the nested two-dimensional hybrid phasedMIMO radar MUSIC peak is clear and complete, accurately and completely estimated 36 targets.

In order to show the accuracy of the estimation more clearly, we compare the root-mean-square error (RMSE) of the three DOA estimates. For these 36 sources, the Monte Carlo number is 100. As shown in Figure 3:

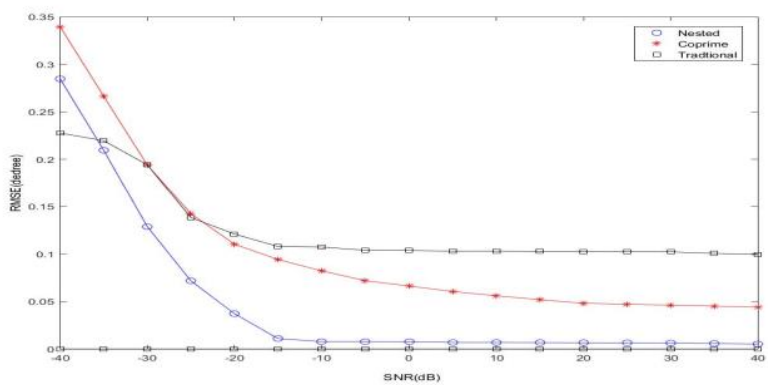

Figure 3 RMSE comparison chart

It can be seen that, except in the environment with extremely low signal-to-noise ratio, the mean square error of the traditional hybrid phased-MIMO radar is slightly smaller than that of the coprime and nested hybrid phased-MIMO radar. As the signal-to-noise ratio increases, the RMSE of this nested two-dimensional hybrid phased-MIMO radar is significantly lower than that of the two-dimensional hybrid phased-MIMO radar.
This is because the difference coarray of "hole-frees" brings great expansion to the virtual aperture, so that the nested two-dimensional hybrid phased-MIMO array can be compared with the traditional and the coprime array, and the number of elements is the same. This nested structure performs less well than the traditional hybrid phased-MIMO radar in low SNR environments, but performs well under high SNR conditions. In this case, there is a more accurate DOA estimation accuracy for the same spatial target. Therefore, it can be considered that the nested two-dimensional hybrid phased-MIMO radar has better DOA estimation performance in a high SNR environment.

\section{Conclusion}

In this paper, a two-dimensional hybrid phased-MIMO radar based on nested array is constructed by using the properties of nested arrays. Furthermore, the array manifold is derived, as well as the position of the array element and the closed expression of the DOF. More importantly, the difference coarray of nested arrays is "hole-free", which maximizes the use of difference coarrays to extend array aperture and DOF compared to traditional and complementary two-dimensional hybrid phased-MIMO radars. A larger aperture and DOF can be obtained given the number of elements, and the DOA estimates better performance.

\section{Acknowledgements}

This work was supported by Project Supported by the National Natural Science Fund of China (61671465).

\section{References}

1. A. Hassanien, S. A. Vorobyov. Phased-MIMO radar: A tradeoff between phased-array and MIMO radars[J]. IEEE Trans. on Signal Processing, 2010, 58(6): 3137-3151.

2. A. Hassanien, S. A. Vorobyov. Y. S. Yoon, et al. Two-stage based design for phased-MIMO radar with improved coherent transmit processing gain $[\mathrm{C}]$ // Signal Processing Advances in Wireless Communications (SPAWC), 2014 IEEE 15th International Workshop on. IEEE, Toronto, ON, Canada: IEEE Press. 2014: 45-49.

3. A. Deligiannis, S. Lambotharan, J. A. Chambers. Beamforming for fully-overlapped two-dimensional phased-MIMO radar[C]. // IEEE Radar Conference (RadarCon), Arlington, VA, USA: IEEE Press. 2015:0599-0604.

4. C. Chen, P. P. Vaidyanathan. Minimum redundancy MIMO radars [C] // IEEE International Symposium on Circuits and Systems. Seattle, WA, USA: IEEE Press. 2008: 45-48.

5. J. Li, D. Jiang, X. Zhang. DOA estimation based on combined unitary ESPRIT for coprime MIMO 
radar[J]. IEEE Communications Letters, 2017, 21(1): 96-99.

6. P. Piya, P. P. Vaidyanathan. Nested array: a novel approach to array processing with enhanced degrees of freedom[J]. IEEE Trans. on Signal Processing, 2010, 58(8):4167-4181.

7. L. Mao, H. Li, Q. Zhang. Transmit Subaperturing for MIMO Radars with Nested Arrays[J]. Signal Processing, 2017, 134: 244-248.

8. M. Yang, L. Sun, X. Yuan, et al. A New Nested MIMO Array With Increased Degrees of Freedom and Hole-Free Difference Coarray[J]. IEEE Signal Processing Letters, 2018, 25(1): 40-44.

9. C. Zhu, H. Chen, Shao H. Joint phased-MIMO and nested-array beamforming for increased degrees-offreedom[J]. International Journal of Antennas and Propagation, 2015: 1-11.

10. S. LIU, B. WANG, X. LI, et al. Design of receiving array of two-dimensional nested hybrid PhasdMIMO radar[J]. Acta Aeronautica et Astronautica Sinic. 2018, 39(4): 173-181.

11. Y. Gu, C. Zhou, N. A. Goodman, et al. Coprime array adaptive beamforming based on compressive sensing virtual array signal[C] // 2016 IEEE International Conference on Acoustics, Speech and Signal Processing (ICASSP), Shanghai, China: IEEE Press. 2016: 2981-2985. 